\title{
AEROSPORAS FUNGICAS DEL AREA ORIENTE DE SANTIAGO (1996-1997)
}

\author{
Fungal aerospores from orient area of Santiago(1996-1997)
}

\author{
Valeria Ibáñez Henríquez, * Gloria Rojas Villegas** \\ *Departamento de Infectología. Facultad de Ciencia Médicas. \\ Universidad de Sanliago de Chile. E-mail mibanez@clx.cl \\ **Departamento de Botánica, Museo Nacional de Historia Natural
}

Palabras clave: Acrosporas fúngicas, alergenos fúngicos acrobiología. Chile.

Key words: Fungal aerospores: fungal allergens. acrobiology: Chile.

\section{RESUMEN}

Utilizando un captador Burkard se estudia la aeromicobiota presente en Santiago oriente durante 12 meses (agosto 1996 a julio 1097) idemificando las aerosporas fingicas por microscopia directa. Estas fueron agrupadas en 19 categorias laxonomicas, correspondiendo el 76.2\% a Deuteromyceles, el 13.6\% a Basidiomyceceles, 2\% a Ascommceles y el 8.3\% a aerosporas no idemificadas:

En muestro regisfro pedominan los hongos demaliáceos debido a la gran presencia de Cladosyorium, 73\%. Las basidiosporas (no idenificadas) constitureron el $8.7 \%$, carbones t. 3\%, ascosporas (no idemificalas) 1.6\%. Alternaria $1 \%$ y el resto de los generos con fiecuencias menores al 1\% Clatosporium presenta un contenido aèreo mavor en otoño (abril) como Epicoccum Stemplylium; las basidiosporas, conidios de Aspergillus/ Penicillium y ascosporas anmentan en los meses de invierno. Alternaria se dispersa con igual frecuencia durante primavera y oloño. Ganoderma y Holminthosporium aumentan durante el rerano.

Con el regisllo de tres años consecutivos se confeccionarà un calendario aeromicológico que permita a los pacientes con rinitis o asma reconocer los periodos de mavor dispersión aérea de estos alérgenos.

\section{INTRODUCCION}

La aerobiología es una herramuienta utilizada al servicio cle otras disciplinas que esıudia las partículas aéreas de origen biológico: virus. bacterias, polen. esporas de hongos. helechos y musgos, pequeñas semillas, protozoos,

\section{SUMMARY}

We studied the existing aeromycobiota in east Santiago for 12 monlhs from august 1996 till july 1997 by using a Burkard rolumetric lrap. The fungal aerospores iclentification was based on their microscopic morphology and they liere classified into 10 taxonomic categories, 76. $2 \%$ correspondiong to Deuteromyceles, $13.6 \%$ Basidiomvciecetes, 2\% to Ascomveles and 8,3\% to unidentified spores. In our survey the Dematiaceous Hyphomyceres prevail due to the great abundance of Cludosporium, 73\% of the total calch. The other most frequent spore lypes were unidentified as basidiospores $(8,4 \%)$, smm $1, \mathrm{~s}(\mathrm{3} \%)$, unidentified ascospores $(1,6 \%)$, Alternaria (1\%). The rest of the fungal spores was observed in small numbers throughout the vear in relative amount loner than 1\%. Cladosporium reached its maximum value in autumm (.April), so did Epicoccum and Stemphylinm. Basidiospores, conidia of AspergillusPenicillium and ascospores increase in winter. Altornaria conidia disperse during spring and autumn with the same fiecuency. Ganoderma and VIelminthosporium increase during the summet:

With the help of three conseculive vears-record it will be possible to prepare an aeromycological calenda. that allow's patient suffering from rhinitis or allergic astlma to recognize the most frequent aerial dispersion limes of lhese allorgens.

ácaros e inseclos. Entre ellas los diversos propágulos de dispersión fúngica constituyen una parte importante de este ambiente (Lacey' 1996).

Poco se sabe en los ambientes externos, de la potencialidad alergénica de las diversas partículas y de los niveles de exposición a que deben estar sometidos los pa- 
cientes para que se les manifieste una respuesta clínica (Levetin et al.,1995). En relación a los hongos, un gran número de especies anemófilas han sido descrilas como agentes desencadenantes de cuadros de asma y rinitis. enlre olras cnfermedades alérgicas respiratorias (Cruz et al. 1997). Cerca de 80 especies han sido asociadas con síntomas de alergia respiratoria, aunque la sensibilización a una sola especie es muy rara: lo habitual, según resultados de pruebas culáneas, es cucontrar reaclividad simullánea Trentc a varios hongos debiclo a la presencia de cpitopes comunes en géneros filogenéticamente relaciomados (Horner et al., 1995).

Mediante encuestas en Santiago (Chile). sc ha detectado en la población infantil, una prevalencia anual de síntomas de asma de un $11 \%$ y de síntomas de rinoconjuntivitis entre 12.7 y $15.7 \%$ (Strachan et al., 1997: Asher el al., 1998).Al mismo tiempo, la reactividad cutánea cs mayor frente a dermatofagoides. polen y polvo de habilación que a las esporas fúngicas (Aguirre et al., 1998: López etal., 1998).

Aunque la concentración atmosféricá de esporas fúngicas, es superior al contenido polínico en nuestra capital (Rojas el al., 1999). como en ol ras partes del mundo. los hongos parecen ocupar un lugar secundario cn la etiología de estos cuadros.

El presente arlículo, analiza los registros mensuaIes obtenidos en una estación de monitorco del aire durante un año, y forma parte de un cstudio a largo plazo y de mayor extensión territorial, que se está realizando en Santiago, con el objeto de confeccionar un calendario esporopolínico que permila a los pacientes alópicos a fectados de estas dolencias, reconocer los períodos de mayor dispersión aérea de estos alergénos inlaalantes y tomar las mediclas adecuadas para disminuir su exposición.

\section{MATERLALES Y METODOS}

Las muestras de aire fueron tomadas desde el I de Agosto de 1996 al 31 de Julio de 1997 en la comuna de Providencia de nuestra capital, con un caplador volumétrico Burkard instalado en el techo de la Clínica Servet; aproximadamente a $15 \mathrm{~m}$ sobre el nivel del suelo. Este aparato funcionó en forma continua durante 7 días. haciendo impactar las partículas suspenclidas en el aire sobre una superficie adhesiva. El aire es succionado a razón de 10 litros/mimuto, simulando el volúmen respirado por un adulto durante aclividad moderada. La cinta adhesiva, de 33.6 $\mathrm{cm}$ de longitud. rola a una velocidad de $2 \mathrm{~mm} / \mathrm{hora}$, de lal modo que fracciones de 48 mm equivalen a un día. En este estudio la cinta Melincx fue cubicrla con vaselina y cambiacla regularmente a las 10 AM los martes de cada semana. Las cintas diarias fueron montadas en una mezcla acuosa de glicerol $(50 \%)$, gelatina (7\%) y fenol ( $1 \%$ ) teñida con
Salfranina y lucgo selladas con cubreobjetos de $5 \times 2,5 \mathrm{~cm}$. La concentración atmosférica diaria fue obtenida cle la lcclura de una banda de $0,45 \mathrm{~mm}$ de diámetro a lo largo de toda la preparación $(4.8 \mathrm{~cm})$, utilizando un ocular de $10 \times$ y objetivo de $40 \mathrm{x}$, que representa el total de aerosporas observaclas en $2+$ horas. El recuento diario fue converlido a conidios/dia/ $/ \mathrm{m}^{3}$ de aire y éste a su vez a cifras mensuales promedio.

La identificación de las aerosporas fúngicas se hizo sólo a nivel de género. mediante rasgos morfológicos microscópicos como color, forma, tamaño y septación, consultando bibliografia especializada (Piontelli \& Toro, 1989; Sunith 199(); Lacaz el al., 1998) y láminas de referencia preparadas a partir de: a) ejemplares de la Micoteca de] Museo Nacional de Historia Natural, b) ejemplares de carbones aportados por el Laboratorio de Micología del Servicio Agricola y Ganadero de Santiago, c) muestras de basidiosporas aportadas por el Prof. Waldo Lazo y d) de hongos anamorfos anemófilos cultivados en nuestro laboratorio.

\section{RESULTADOS Y DISCUSION}

En las 365 muestras colectadas durante un periodo anual, se contaron un tolal de 987.516 conidios y/o esporas füngicas (coleclivamente clenominadas aerosporas), las que fueron agrupadas en 1.3 taxa $y 6$ categorias. Los Deuteromycetes constituyeron el $76.2 \%$ del tolal de ellas, los Basiliomycetes al 13.6\%, los Ascomycetes el 2\% y cl $8.3 \%$ no identificado se clasificó como dematiáceo o hialino según presencia o ausencia de pigmento oscuro. Si bien los Deuteromycetes, fundamentalmente los Myplıomycetes dematiáceos predominan en la mayoría de los monitoreos atmosféricos en diversas partes del mundo, su concenl ración relativa es variable, fluctuando entre el 40 al $80 \%$. En la atmósfera de Ciudad de México y en la de Waterloo (Canadá), Ias aerosporas de Basidiomycetes han sido el componente mayor, represcntando el 30 y $24 \%$ respeclivamente. En ambas ciudades las ascosporas también constituyen una fracción mayor a la obtenida en nuestro estudio, 12 y 10\% (Calderón et al., 1995: De-Wei \& Kendrick $.1995)$.

La concentración media tolal anual registrada en Santiago oriente fue de 2.706 aerosporas/día/ $\mathrm{m}^{3}$ con valores que fluctuaron entre 192 y 10.315, esta última ocurrida en Abril. Al parecer. las condiciones climáticas y disponibilidad de sustratos adecuados presentes cntre Marzo y Junio, resultaron favorables para la liberación, clispersión y conidiogénesis de los hongos dematiáceos predominanics, por cuanto la mayor concentración aérea se observó en otoño, estación que concentró el $37 \%$ del lotal de lats aerosporas. El contenido fúngico en el resto de las cstaciones, no presentó variaciones muy importantes, flucluan- 
Tabla 1.- Aerosporas fúngicas de Santiago oriente (Conidios/día/m³)

\begin{tabular}{|l|c|c|c|c|}
\hline \multirow{2}{*}{ Taxa-categoría } & \multicolumn{3}{|c|}{$1996-97$} \\
\cline { 2 - 5 } Cladosporium & $\begin{array}{c}\text { Frecuencia } \\
\text { relativa } \%\end{array}$ & $\begin{array}{c}\text { Media } \\
\text { anual }\end{array}$ & $\begin{array}{c}\text { Recuento } \\
\text { máximo }\end{array}$ & $\begin{array}{c}\text { Alergeni- } \\
\text { cidad }\end{array}$ \\
\cline { 2 - 5 } Basidiosporas no id. + & 8,4 & 228 & 1120 & $*$ \\
Carbones & 4,3 & 117 & 1978 & \\
Ascosporas no id. & 1,6 & 43 & 238 & $*$ \\
Alternaria & 1.0 & 27 & 168 & $*$ \\
Aspergillus/Penic. & 0,8 & 22 & 459 & $*$ \\
Ganoderma & 0,7 & 18 & 87 & $*$ \\
Stemphylium & 0,4 & 11 & 132 & $*$ \\
Helminthosporium & $*$ \\
Epicoccum & 0,4 & 11 & 67 & $*$ \\
Torula & 0,3 & 7 & 48 & $*$ \\
Chaetomium & 0,3 & 8 & 42 & $*$ \\
Royas & 0,3 & 7 & 36 & $*$ \\
Pleospora & 0,2 & 6 & 62 & \\
Oidium & 0,1 & 2 & 44 & \\
Polythrincium & 0,1 & 1 & 24 & \\
Curvularia & 0,02 & 1 & 11 & \\
Dematiáceos & 0,02 & 1 & 14 & \\
Hialinos & 3,9 & 103 & 495 & $*$ \\
\hline
\end{tabular}

* Complex de especies semejinte, Incluye conidios compatible con Exerohilum, Bipolaris y Drechslera.

+ no idl. $=$ no iclentiliciddss

do entre el 18 y 2 \% $\%$. Un patrón estacional similar, de mayor presencia en otoño, pero que comienza a finales clel verano. se ha informado en latitudes scmejantes a la nues(ra como en Brisbane y: Melbourne (Australia) (Rulherford et al., 1997: Milakakis e' al., 1997). donde cl 66\% del contcniclo se registró durante los meses de verano-otoño. Un Cranco predominio en verano ocurrió en Waterloo (op cil.) ciudad que registra una media anual de 3.477 aerospora/ dia/ $/ \mathrm{m}^{3}$, superior a la nuestra. En un monitoreo atmosférico realizado cn 199]-92 cn Santiago norte. la mayor concenuación se obluvo en verano (Jbáñez et al. 1998).

La Tabla 1, muestra las aerosporas iclentificadas en

cste cstudio. Sus čoncentraciones medias, cifras máximas y su contribución relativa al recuento total; además se inclican aquellos géneros fímgicos en los que se ha demostrado alergenicidad por su reactividald cutánea. por delección sérica de IgE específicas o por pruebas de provocación (Kozak \& Hoffman, 1984; Cruz et al., 1997). En la Figura I se ilustra la dinámica aérca de los principales hongos deteclados en la atmósfera de Santiago oriente expresada con sus media mensuales.

Destaca la marcada dominancia de Cladosporium en nuestra atmósfera que constituye el $73 \%$ del contenido fúngico en este período, con una media anual de 1.975 


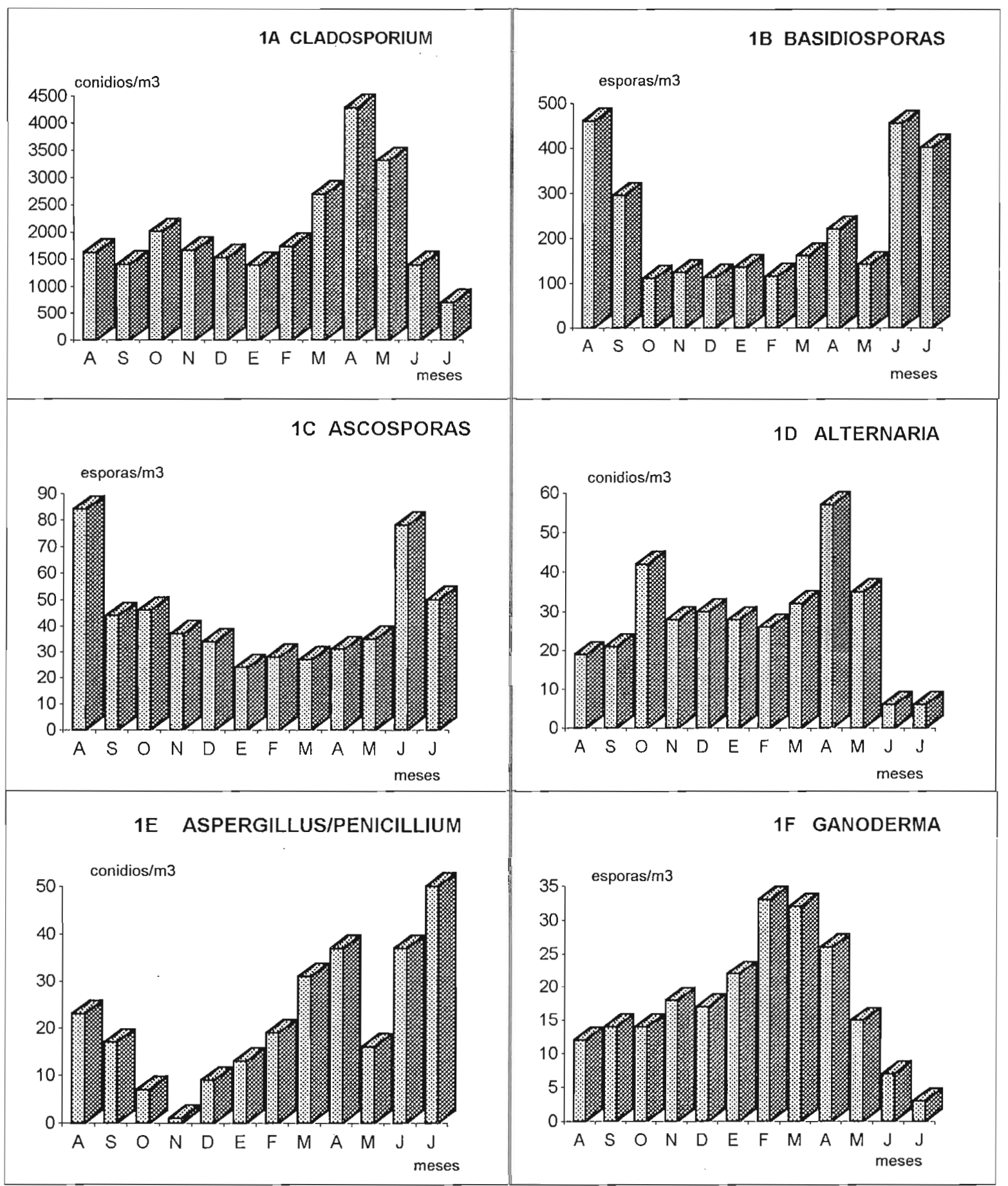

Figura 1. Dinámica de aerosporas fungicas alergénicas Santiago oriente 1996-97 
conidios $/ \mathrm{dia} / \mathrm{m}^{3}$, con cifras diarias que fluctuaron entre 64 y 9.240 (Abril). Su período de mayor dispersión ocurrió en Abril y Mayo, lo que determinó que en oloño se captó la mayor cantidad de conidios $/ \mathrm{m}^{3}$ de estos propágulos y el menor contenido en invierno (Fig. IA). Las frecuencias de recolección informadas en la literatura fluclúan entre 35 y $75 \%$ y en general el contenido atmosférico es mayor durante el verano y' cl oloño, lo que nos ubica dentro de este contexto (Herrero \& Zaldivar, 1997; Waissel el al. 1997).

Kurkela (1997), señala que el crecimiento, producción de conidios y dispersión de Cladosporium, se correlaciona positivamente con el aumento de la temperalura, disminución de la humedad relativa y sólo con el pcriodo inicial de la lluvia, la que permitc el desprendimiento de los conidios de su estructura de fructificación. Estos datos son congruentes con lo encontrado previamente por Tbáñcz el al., (1998).

Cladosporium es el principal componente de la micobiola en la mayoría de las investigaciones aerobiológicas, con sumas anuales que fluctuan ampliamente, como la sido registrado en Holanda en observaciones diarias, cuyas cifras máximas oscilaron entre 450.000 y 1.895 .000 por $\mathrm{m}^{3}$ (Nikkels et al., 1996). En nuestro estudio la suma anual fue de 720.838 conidios $/ \mathrm{ml}^{3}$.

Las basidiosporas ocupan el segundo lugar en orden de frecuencia, con un $8.4 \%$ y una media anual de 228 esporals/dia/ $/ \mathrm{m}^{3}$. con fluctuaciones diarias entre 24 y 1.120 por III $^{3}$. Este grupo heterogénco incluye esporas de Coprinus, Agaricus, Boletus, Calvatia y Agrocybe, jun10 a un gran número de esporas no identificables a nivel de género. tanto hialinas como dematiáceans. En la Fig. IB se ilustra el patrón bimodal de esta categoría. caracterizado por una concentración baja de octubre a mayo, lanqueado por dos períodos de mayor dispersión de similar magnitud. Las basidiosporas se detectaron principalmente en los meses fríos y de mayor humedad: en invierno se concentró el $43 \%$ del tolal recopilaclo en el año y en onoño el $28 \%$, mientras en primavera y verano se distribuyeron en forma similar $(15 \%)$. En otras partes del mundo la estacionalidad de este grupo es similar o aumentan durante el otoño (Horner el al., 1998). Las esporas de Coprinus son las basidiosporas aerotransportadas más comunes vistas en este estudio. al igual que lo observado en Ciudad cle México por Calderón é al., (1995). Estos autores encontraron correlación direcla entre la concentración aérea cle basidiosporas y humedad relativa $(>7(0 \%)$ y lambién con la IJuvia. cuando ésla cae 2 a 4 días antes de la medición, sugiricndo que es el ticmpo requerido por algunos basicliocarpos para su maduración. En relación a la concentración diaria señalan que en zonas urbanas, raramente sobrepasan lás 2.000 por $\mathrm{m}^{3}$, pero en áreas rurales o boscosas, pueden llegar a $100.000 / \mathrm{m}^{3}$ por corlos períodos de tiempo y constituir en ellas hasta el 70\% del tolal de las aerosporas registradas. Han sido caracterizados como alergénicos Boletus, Calvatia, Coprinus, Ganoderma, Pleurotusy Psilocybe (Horner et al., 1998).

Los carbones pertenecen al orden Ustilaginales y son las estructuras de dispersión anamórficas de estos Basidiomycetes. La gran mayoría de ellos son parásitos de plantas, pastos y cereales. Los conidios de Ustilugo y Tilletia, son los que observamos con mayor frecuencia. En nuestro registro ocupan el tercer lugar en orden de frecuencia. Si los carbones juegan algún rol en la alergia respiratoria éste aún no está determinado (Horner et al., 1995).

Las ascosporas son otro gran grupo de propágulos que tienen importancia en los registros aerobiológicos, tanto por su concentración como por la capacidad alergénica que presentan algunos géneros. En Santiago oriente constituyeron el $1.6 \%$ del contenido atmosférico y la suma anual regislrada por el grupo fue de $15.769 \mathrm{esporas} / \mathrm{m}^{3}$. Predominaron en invierno (37\%), mientras en otoño y primavera sumaron el $50 \%$ de ellas, disminuyendo en los meses calurosos (Fig. 1C). En la literatura europea se informan frecuencias de recoleción con rangos del 5 al 20\% y sumas anuales de la concentración diaria de hasta 15.000 esporas por $m^{3}$ de aire (D'Amato, 1995). Las ascosporas agrupadas en esta categoría en nuestro estudio, son compatibles con Leptosphathia, Paraphaeosphaeria, Sporormiella, Xilaria y Massarina, entre otras. Algunos géneros que han sido asociados a enfermedades alérgicas son Leptosphacria, Xilaria, Chaetomium (Cruz et al., 1997).

Los conidios de Alternaria, fueron la quinta frecuencia registrada, aportando solo el $1.0 \%$ del total del contenido atmosférico en el período 1996-97, con una media anual de 27 por $\mathrm{m}^{3}$ y una máxima diaria de 168 . Presentó dos períoclos de mayor dispersión aérea cuyas máximas ocurrieron en los meses de octubre y abril (Fig. ID), con mayor frecuencia en primavera $(32 \%)$ y otoño $(33 \%)$; y solo un $11 \%$ en invierno. Unal estacionalidad algo diferente se ha registrado en Sydney. con predominio en primavera y verano y cifras diarias máximas de 1.125 por $\mathrm{m}^{3}$ ( diez veces mas allas que las nuestras), sin embargo, las sumas anuales fueron moderadamente mas allas que nuestros registros ( 16.600 por $\mathrm{m}^{3}$ versus 10.025 de Santiago) (Bass \& Morgan, 1997). En Melbourne, el verano concentra entre el 37 al $47 \%$ del tolal de estos conidios y sus sumas totales anuales no superaron los 7.000 (Mitakaki et al., 1997) Ambos estudios australianos se prolongaron por tres años consecutivos, entre 1992 y 1995. En Holanda (op. cit.) las sumas anuales de este taxón siempre resultaron mas altas que las observadas en esle estudio, entre 10.735 y 31.580 , concentrándose también en la estación estival. En Palencia(España), Alternaria, representa el 9\% del total de aerosporas impactadas, logrando su mayor concentración en verano y sus medias semanales no sobrepasan las 80 por $\mathrm{m}^{3}$ (Herrero \& Zaldivar, 1997). 
Los conidios de Aspergillus/Penicillium. reconociendo como tales a todas aquellos propágulos unicelulares lialinos que se presentaron en cadenas, conformaron el $0,8 \%$ de la micobiola anemófila. con una media anual de 22 por $\mathrm{m}^{3} \mathrm{y}$ una suma anual de 7.915: registraron mayor presencia durante el otoño-invierno (79\%). disminuyendo notablemente en primavera (Fig. IE). Estos son lo propágulos de mas difícil reconocimicnto, tanto por su pequeño tamaño como por su carácler incoloro, y junlo con las basidiosporas de los Lycoperdales («punf-ball»), son a nuestro juicio, los más subestimados con nuestra metodología. En la revisión efectuada por D'Amato \& Spickman (1995). se señala que cn Europa, su presencia constituy'c entre el 2 y $20 \%$ del contenido total y que en el año pueden llegar a sumar hasta 15.000 conidios por $\mathrm{m}^{3}$, cifras superiores a las nuest ras.

Las basidiosporas de Ganoderma, muy distintivas y fáciles de reconocer en las preparaciones direclas, presentaron una dinámica aérea caracterizada por solo un período de mayor dispersión, el que comienza paulatinamente en noviembre hasta lograr un peak cn febrero-marzo y disminuir fuertemente en los meses fríos (Fig. IF). Constituyó el $0.7 \%$ del contenido fúngico tolal, logrando una media anual de 18 por m $^{3}$, una cifra incividual máxima de 87 y una suma anual de $6.45+$ esporas por $\mathrm{m}^{3}$. En verano se concentra el $38 \%$ de su contenido, distribución estacional similar a la observada en Ciudad cle México y Waterloo. aunque en el monitoreo realizado en esta última ciudad alcanzaron una frecuencia relativa de $7.2 \%$ y una media anual de 248 por $\mathrm{m}^{3}$, muy superior a la nuestra.

Los otros hongos identificados a nivel de género en este estudio contribuyeron con un porcentaje menor al $0,5 \%$ del contenido total. La suma anual alcanzada por Stemphylium fue de 4.136 conidios por $\mathrm{m}^{3}$, seguido por el complex Helminthosporium con 4.085, Torulu con 2.748 , Chatomium con 2.715 y Epicoccum con 2.55t. Sumas anulales menores de 600 por $\mathrm{m}^{3}$ presentaron las acrosporas de Pithomyces, Curvularia, Polythrincium, Oidium Pleospora. Salvo Epicoccum que en Waterloo alcanzó una media anual de 46 conidios por $\mathrm{m}^{3}$ y una frecuencia de $1.3 \%$, el resto de los hongos enumerados también tienen escasa representación en csa atmósfera canadiense. Epicoccum fue más abundante en Leiden (Canadá) cuyas sumas anuales en promedio alcanzan las 6.629; Stemphylium en cambio, registra sumas promedio menores, de 1.100.(Nikkels et al., 1996). Epicoccum, Stemphylium y Curbularia tienen concentraciones mas altas durante el verano, en primavera en cambio lo hacen las aerosporas de royas, Torula, Polythrincium, Fusicladium, Oidium y Pleospora.

\section{CONCLUSIONES}

La metodología utilizada en este estudio fue desarrollada inicialmente para estudiar el componente polínico, hacienclose extensivo también para los hongos. La principai ventaja de este método sobre el del cullivo, es que aumenta cl rango de propágulos que pueden ser cuantificados y su aspiración contínua permite además determinar la fluctuación horaria. Su principal desventaja radica en la dificultad de identificar las aerosporas de morfología similar, que requieren de la observación de sus estructuras de fruclificación especiamente Deuteromycetes hialinos o dematiaceos como Aspergillus, Penicillium, Fusarium, Scopulariopsis, entre otros y muchas basidiosporas.

Sin embargo. con diferentes metódicas los conidios de Cladosporium son lẹos, el principal componente de la micobiola atmosférica en Santiago y otras latitucles. Cladosporium forma parte del gran número de géneros fúngicos anamórlicos que han sido descritos como agentes sensibilizadores en desórdenes alérgicos. De los hongos aislados en nuestro estudio, se han caracterizado algunos alergénos en Cladosporium, Alternaria, Aspergillus, Epicoccum, Fusarium, Helminthosporium, Penicillium y Botrytis.

La concentración de las basidiosporas es alta en Santiago, sin embargo, puede estar subestimada, especialmente las más pequeñas $y$ hialinas, por la gran cantidad de material particulado suspendido en la atmósíera, principalmente en Jos meses de invierno, donde predominan (está dificultad se hace extensiva a todos los conidios pequeños). Esto explicaría que entre el 25 y $30 \%$ de las personas con alergia respiratoria (asma). están sensibilizadas al alguna especie de Basidiomycetes (Horner, 1998). De ellas, Coprinus y Ganoderma son componentes importantes en nuestra micota anemórila.

Debido a las fluctuaciones estacionales que presenta año a año la concentración aérea de los hongos, es necesario un calendario micológico a tres años mínimo, para predecir su comportamiento y lomar las medidas de prevención.

\section{FINANCIAMIENTO}

Este trabajo fue financiado por FONDECYT p 1960005.

\section{REFERENCIAS}

Aguirre, V.; Mallol, J.; Sempertegui, F.; Kovacevic, V. (1998) Antilisis descriptivo del Test Cutineo ell el estudio del sindrome bronçuial obstructivo en niños. Rev Chil Enf Respir: $14: 232$
Asher, M.; Anderson, H.; Stewart, A.; Crane, J. (1998). Worldwicle valiations in the prevalence of asthma symptoms: the Intemational Sudy of Asthma and Allergies in Childhood (ISAAC). 
Eur Respir I:12:315-33,5

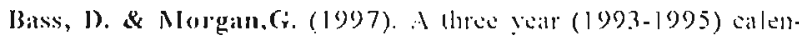
dar ol pollen and Allermaria mould in the atmosplere ol south western Sivales: (irana .36: 293-300

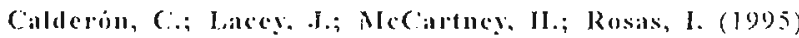
Seasonal and dimal variation ol airborme basidiomyese sjome conconlmitums in N/sxico (ity. Giana $34: 260-268$

C'ruz, 1.; Saenz de Santantaria, M.; Maltinez, J.; Martiney A.: (iuisantes, J.; Palacios, R. (1997) fiungal allergens liom importan allergenic lingi imperfecti. Allcrgol el lmmunopathol $25: 153-58$

Ja Silva, C.; Porto, li.; Ileins- Yaccari, Ri., Takoluashi, N. (1998)

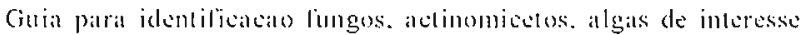
medico. Eal. Sarvior Siro Paulo, Brisil.

D'Amato, (i. \& Spicksma, fi. (1995) Aerobiologic and clinical aspects ol mould allergy in liorops. Allergy 50:87()-77

De-Woi, i. \& liendrick, B, (1995). A year-round ondoor arromycological study in Walcrloo. Ontario. Camadil. Grama 34: $199-297$

Ilerrero. B. \& 7aldivar; P. (1997). Fillects of" melcological litcturs on the levels of slemaria and Claclosporim spores in the atmusplere ol Palencial. 1990-92. Cirana 36:180-18.t

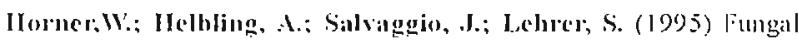
allergons. (lin. Microbiol. Ro. $\$: 161-79$

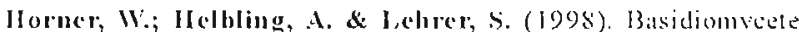
allergens. Allergy $53: 111+-1121$

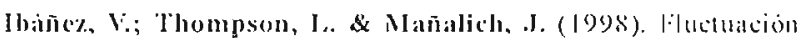
estacional de hongos ancmulilos sn Santiano Norts - Chile. Bol. Nicol. 13:47-56

Kotak, .J. \& lloflman, l). (19\$4) Critical review ol slitgnoslic procedure lor mould allergy: lin slould Allergs: Fid. Al-Doory Y. and

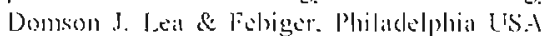

Kurkela, T'. (1997). "The number ol" Cladosporium conidia in the air in dillerent weather conditions. Grana 36:54-61

lacey. I. (1996). Spore dispersal-it role in ecology an discase: the Britikh contribution to fungal atrobiolog: Mycol.Rex. 100:6+1660
Levetin, E.;Shaughnessy, R.; Fischer,L.; Ligman, B.; Ilarrison, J.; Bremun, $\Gamma$. (1995). Indoor air quality in a Schools: exposute tu lingal allergens. Aerobiol. 11:27-34

I.opez, M.; Parada, O. \& Feijoo, R.M. (1998) Test cutaneo : revision de 2024 eximenes. Institule Nacional del lörax. Rextmen. Rev. Chil. Ent. Rexpir. 14:234

Mitakakis, T.; Kog Ong, li., Stevens, A.; Guest, D.; Bruce, R. (1997) Incidence of Cladosporium. Altemaria and tolal lingal spores in the almosphere of Malbourne (Australia) over thes years. Acrobiologia 1.3:8.3-90

Nikkels, A.; Terstegge, P. \& Spicksma, li. (1996). Ten 1ypes of microscopically identiliable airborne limgal spores at Leiden. "lho Netherlans. Acrobiologia 12:107-112

Piontelli, li. \& loro, M. (1989) Introduccion al estudio de los microlungos. Guia de identification generica. Parte I: Macorales. Ascomyceles. Dedteromicoles. Fac. Medicina. Iniversidad de valparaiso.

Rojas, (;.; Roure, J.M.; Galleguillos, F.; Mardones, P. (1999). Aeropalinulogias de Simtiago. Rev. Chil. Enl. Respir. 15:1+1-155

Rutherford, S.; Owen, J. \& Simpson, R. (1997). Survey of airspore in Brisbane, Quesnsand. Australia. Grana 36:114-121

Strachan, D.; Sibbald, B.; Weiland, S.; Ait-Khaled, N.; Anabwan, G.; Anderson, Il.; Asher, M.; Beasley, R.; l3jorkstén, I3.; Burr, M.; C.Iayton, 'Г.; C:rane, .J.; Fllwood, P.; Keil, U.; Lati, C.: Mallol, J.; Maltinez, IF; Mitclıell, E.; Montefort, S.; Pearee, N.; Robertson, C.; Slual, .J.; Stewart, A.; von Mutius, li.; Williams, II. (1997) Wiorldwide vallations in prevalenes of symptoms of allergic rhinoconjunctivitis in children: the International Study of Asthma and Allergies in Childhood (IS: A.tC). Pediall, Allergy: Inmmol. 8:16/-176

Smith, E.C: (1900) Sampling and identilying allergenic pollens and moulcls. Allas. $2^{\circ}$ ed. Blewstone Press. San Antonio. USA.

Waisel, V.; Camor, li.; Glikman, N.; Epstein, Vi; Brenner, S. (1997). Airborno liungal spores in the coastal plain of Isratel a preliminary surey. Acrobiologia 13:281-287 